\title{
Association of blood type A with increased risk of severe COVID-19 in healthcare workers
}

\author{
Niraj N. Mahajan ${ }^{1} \cdot$ Rahul K. Gajbhiye $^{2} \cdot$ Periyasamy Kuppusamy $^{2} \cdot$ Shubhada Bahirat $^{1} \cdot$ Pradip D. Lokhande $^{1}$
}

Received: 6 July 2021 / Accepted: 16 August 2021 / Published online: 22 August 2021

(c) The Author(s), under exclusive licence to Springer-Verlag GmbH Germany, part of Springer Nature 2021

\section{Dear Editor,}

We have read with great interest the article by Latz et al. [1] reporting no association of blood type with risk of progression to severe COVID-19 requiring intubation or causing death. Maharashtra is among the worst affected states in India during the ongoing second wave of the COVID-19 pandemic. The second wave of the COVID-19 outbreak has been ongoing in Mumbai since February 2021, with 30-40 newly confirmed moderate to severe cases reported per day at BYL Nair Charitable Hospital (NH), a dedicated COVID-19 hospital in Mumbai, India. The exponential rise of COVID-19 cases and higher deaths could be due to the new variant of SARS-CoV-2 (B.1.617) which is now classified as variant of concern [2]. For combating the SARS-CoV-2 infection, healthcare workers (HCWs) have been on the forefront making them vulnerable for a higher risk of contagious SARS-CoV-2 infection.

Identification of risk factors for increased susceptibility for COVID-19 in healthcare workers is urgently required especially during the devastating second wave to reduce the mortality and also plan the policies for HCWs. Toward this, ABO gene has been reported as one of the risk factors for COVID19-related mortality and morbidity with emerging evidence on blood type having a role in COVID-19 susceptibility. ${ }^{2}$ Some of the studies reported the association of ABO blood

Niraj N. Mahajan MD and Rahul K. Gajbhiye contributed equally to this work.

Trial registration: The study is registered with Clinical Trial Registry of India (Registration No.: CTRI/2020/09/027516 dated 01/09/2020). Retrospectively registered.

Niraj N. Mahajan

nirajdr@hotmail.com

1 Department of Obstetrics and Gynecology, Topiwala National Medical College \& BYL Nair Charitable Hospital, Mumbai 400008, India

2 Clinical Research Laboratory, ICMR-National Institute for Research in Reproductive Health, Mumbai 400012, India group polymorphism linked with COVID-19 susceptibility and adverse clinical outcomes [3, 4]. However, the results of these studies were contradictory suggesting more evidence from different populations and healthcare settings to be generated. Considering this urgent need, we studied the relationship of severity and virus clearance (VC) of COVID-19 with blood type among HCWs. The data was captured from the medical case records and interviews of HCWs with COVID-19 from 6 April to 31 October 2020. VC was calculated from testing positive for SARS-CoV-2 infection to two successive negative swabs $48-72 \mathrm{~h}$ once patient became asymptomatic.

There was a notable difference among HCWs with SARS$\mathrm{CoV}-2$ infection when their $\mathrm{ABO}$ blood groups were compared with frequency in Indian population [5]. Of the $491 \mathrm{HCWs}$ with COVID-19, ABO blood type was available for $367 \mathrm{HCWs}$. The frequency of HCWs who had SARS-CoV-2 infection with type $A$ and $A B$ group was significantly higher than that in the general population (type A: 0.038 ; type $\mathrm{B}: p=0.002$ ), while the frequency of HCWs with type $\mathrm{O}$ type was significantly lower than the general population $(p<0.001)$ (Table 1$)$. There is $36 \%$ higher chance of more severe disease in blood types $\mathrm{A}$ and $\mathrm{AB}$ than in B and O. Risk of SARS-CoV-2 infection is less with blood type "O" [OR 0.63 (95\% CI 0.49-0.80)], whereas it is more with type AB [OR 1.67 (95\% CI 1.21-2.32)] and type A [OR 1.29 (95\% CI 1.02-1.64)] (Supplementary Tables 1 and 2). VC analysis was done for $357 \mathrm{HCWs}$ with available blood type. There was no significant difference in VC among the different blood types among HCWs $(p=0.792)$ (Table 1$)$.

Our results demonstrate the effect of $\mathrm{ABO}$ blood group on SARS-CoV-2 infection and disease severity in HCWs in India. We observed that $\mathrm{HCW}$ s with blood groups $\mathrm{A}$ and $\mathrm{AB}$ had an increased risk for COVID-19 similar to other studies $[3,4]$ and blood type $\mathrm{O}$ was associated with a reduced risk similar to the studies reported in the literature $[3,4]$. All the HCWs recruited in this study represent the first wave of the COVID-19 pandemic in India. We did not look at the relationship between SARS-CoV-2 virus strain and blood group which is a limitation of our study. SARS-CoV-2 receptor 
Table 1 ABO blood type frequency and its relationship with remission, risk, and severity of COVID-19 among HCWs

\begin{tabular}{llllll}
\hline Parameters & \multicolumn{2}{l}{ ABO blood type } & \multirow{2}{*}{$p$ value } \\
\cline { 2 - 5 } & $\mathrm{A}$ & $\mathrm{B}$ & $\mathrm{AB}$ & $\mathrm{O}$ & \\
\hline HCWs with COVID-19 $(n=367)$ & $101(27.5)$ & $119(32.4)$ & $45(12.3)$ & $102(27.8)$ & $<0.001$ \\
Reference blood type $^{\mathrm{a}}(n=9686)$ & $2216(22.9)$ & $3125(32.3)$ & $750(7.7)$ & $3595(37.1)$ & \\
$p$ value $^{\mathrm{b}}$ & 0.038 & 0.948 & 0.002 & $<0.001$ & \\
Remission $(n=357)$ & $99(27.7)$ & $117(32.8)$ & $44(12.3)$ & $97(27.2)$ & $<0.001^{*}$ \\
Virus clearance in days $(n=357)$ & $10(7-14)$ & $11(6-14)$ & $10(7-13)$ & $11(6-14.5)$ & 0.792 \\
Duration of illness to recovery in & $13(10-17)$ & $14(10.5-17)$ & $13(10.5-15.5)$ & $13(9-16.5)$ & 0.863 \\
$\quad$ days $(n=357)$ & & & & & \\
Severity of disease $(n=367)$ & $101(27.5)$ & $119(32.4)$ & $45(12.3)$ & $102(27.8)$ & $<0.001^{*}$ \\
Asymptomatic $(n=41)$ & $11(10.9)$ & $15(12.6)$ & $3(6.7)$ & $12(11.8)$ & 0.750 \\
Symptomatic $(n=326)$ & $90(89.1)$ & $104(87.4)$ & $42(93.3)$ & $90(88.2)$ & \\
Mild $(n=270)$ & $78(77.2)$ & $84(70.6)$ & $33(73.3)$ & $75(73.5)$ & $0.616^{\#}$ \\
Moderate $(n=49)$ & $10(9.9)$ & $17(14.3)$ & $8(17.8)$ & $14(13.7)$ & \\
Severe $(n=7)$ & $2(2.0)$ & $3(2.5)$ & $1(2.2)$ & $1(1)$ & \\
\hline
\end{tabular}

Categorical variables are represented as number (percentage) and compared using the chi-square test. Continuous variables are represented as median (interquartile range) and compared using the Kruskal-Wallis test

${ }^{\text {a}}$ Represents reference blood type of Indian population (Agarwal et al.) [5]

${ }^{b}$ Each blood type compared to all other blood types of HCWs with COVD-19 controlled with reference blood type of Indian population

* Comparison was made with reference blood type of Indian population

${ }^{\#}$ Comparison was made between mild vs moderate and severe binding domain's preference of recognizing and attachment to the blood type A antigen was found in the respiratory epithelial cells [4] of HCWs that may provide a greater insight into the potential link between blood group A and COVID19 disease. Therefore, we recommend that HCWs with blood type A should take utmost precautions while working in high-risk exposure areas of SARS-CoV-2.

Supplementary Information The online version contains supplementary material available at https://doi.org/10.1007/s00277-021-04641-6.

Author contribution Niraj N. Mahajan: conceptualization, data curation, formal analysis, methodology, project administration, resources, supervision, validation, writing original draft, and review and editing.

Rahul K. Gajbhiye: conceptualization, methodology, writing original draft, and review and editing.

Periyasamy Kuppusamy: statistical analysis, and review and editing.

Shubhada Bahirat: resources, acquisition of data, and review and editing.

Pradip Lokhande: resources, acquisition of data, and review and editing.

Data availability Data available on request due to privacy/ethical restrictions.

Code availability Not applicable.

\section{Declarations}

Ethics approval The study was approved by the Ethics Committees of TNMC (No. ECARP/2020/78 dated 13.08.2020).
Consent to participate and for publication The information on COVID-19 exposure was collected on telephonic interviews. All HCWs gave informed consent and agreed for the anonymous use of their medical data for publication.

Conflict of interest The authors declare no competing interests.

\section{References}

1. Latz CA, DeCarlo C, Boitano L et al (2020) Blood type and outcomes in patients with COVID-19. Ann Hematol 99:2113-2118. https://doi.org/10.1007/s00277-020-04169-1

2. Weekly epidemiological update on COVID-19 (2021) https:// www.who.int/publications $/ \mathrm{m} /$ item/weekly-epidemiologicalupdate-on-covid-19---4-may-2021. Accessed July 5, 2021

3. Wu Y, Feng Z, Li P et al (2020) Relationship between ABO blood group distribution and clinical characteristics in patients with COVID-19. Clin Chim Acta 509:220-223. https://doi.org/10.1016/j.cca.2020.06.026

4. Wu SC, Arthur CM, Wang J et al (2021) The SARS-CoV-2 receptor-binding domain preferentially recognizes blood group A. Blood Adv 5:1305-1309. https://doi.org/10.1182/bloodadvan ces. 2020003259

5. Agrawal A, Tiwari AK, Mehta N et al (2014) ABO and Rh (D) group distribution and gene frequency; the first multicentric study in India. Asian J Transfus Sci 8:121-125. https://doi.org/ 10.4103/0973-6247.137452

Publisher's note Springer Nature remains neutral with regard to jurisdictional claims in published maps and institutional affiliations. 